\title{
Description of a new species of Paracrobeles Heyns, 1968 (Nematoda, Rhabditida, Cephalobidae) from Kelso Dunes, Mojave National Preserve, California, USA
}

\author{
Sven BOSTRÖM ${ }^{1} \&$ Oleksandr HOLOVACHOV ${ }^{2}$ \\ ${ }^{1,2}$ Department of Zoology, Swedish Museum of Natural History, Box 50007, \\ SE-104 05 Stockholm, Sweden. \\ ${ }^{1}$ E-mail: sven.bostrom@nrm.se (corresponding author) \\ ${ }^{2}$ E-mail: oleksandr.holovachov@,nrm.se \\ ${ }^{1}$ urn:Isid:zoobank.org:author:528300CC-D0F0-4097-9631-6C5F75922799 \\ ${ }^{2}$ urn:Isid:zoobank.org:author:89D30ED8-CFD2-42EF-B962-30A13F97D203
}

\begin{abstract}
A new species of Paracrobeles, P. kelsodunensis sp. nov. is described from the Kelso Dunes area, Mojave National Preserve, southern California. Paracrobeles kelsodunensis sp. nov. is particularly characterised by a body length of 469-626 $\mu \mathrm{m}$ in females and $463-569 \mu \mathrm{m}$ in males; lateral field with four incisures, extending almost to tail terminus; three pairs of asymmetrical lips, separated by U-shaped primary axils with two long guarding processes, each lip usually with four tines along its margin; three long labial probolae, deeply bifurcated, with slender prongs without tines; metastegostom with a strong anteriorly directed dorsal tooth; pharyngeal corpus anteriorly spindle-shaped, posteriorly elongate bulbous with dilated lumen; spermatheca 24-87 $\mu \mathrm{m}$ long; postvulval uterine sac 60-133 $\mu \mathrm{m}$ long; vulva in a sunken area; spicules 33-38 $\mu \mathrm{m}$ long; and male tail with a 5-8 $\mu \mathrm{m}$ long mucro. The generic diagnosis is emended on the basis of recently described species and a key to the species of Paracrobeles is provided.
\end{abstract}

Keywords. Morphology, new species, Paracrobeles, SEM, taxonomy.

Boström S. \& Holovachov O. 2015. Description of a new species of Paracrobeles Heyns, 1968 (Nematoda, Rhabditida, Cephalobidae) from Kelso Dunes, Mojave National Preserve, California, USA. European Journal of Taxonomy 117: 1-11. http://dx.doi.org/10.5852/ejt.2015.117

\section{Introduction}

The genus Paracrobeles was erected by Heyns (1968), with Paracrobeles laterellus Heyns, 1968 as type species, for a cephalobid from South Africa having: pharyngeal metacorpus developed into an elongate median bulb with a large chamber; labial probolae long, bifurcate, sharply pointed, prongs without fringe; and cephalic probolae prominent, sharply pointed without fringe. P. laterellus has also been reported from Namibia by Rashid et al. (1990). Since then three new species have been described, viz. P. psammophilus Navarro \& Lluch, 1999 from Spain, P. mojavicus Taylor, Baldwin \& Mundo-Ocampo, 2004 from California, USA and P. deserticola Abolafia, Divsalar, Panahi \& Shokoohi, 2014 from Iran. P. psammophilus was also recorded from Italy by Orselli \& Vinciguerra (2002), who presented SEM 
pictures of the species. A population of Paracrobeles from Kelso Dunes, Mojave National Preserve, southern California, is described here from studies by light and scanning electron microscope. It is close to P. mojavicus, but differs in some characters and is thus considered to represent a new species. The generic diagnosis is emended on the basis of recently described species and a key to the species of Paracrobeles is provided.

\section{Materials and methods}

During a tour in the Kelso Dunes area, Mojave National Preserve, southern California, the junior author collected several samples of sand in the rhizosphere of different species of desert plants along the southern slope of the dunes. Nematodes were recovered from sand samples using a modified Baermann funnel extraction technique. For LM, specimens were relaxed by gentle heat, fixed in cold $4 \%$ formaldehyde solution, transferred to pure glycerine by a slow evaporation method and mounted on permanent slides in glycerine with paraffin wax as support for the coverslip. For SEM, specimens were post-fixed in $1 \%$ osmium tetroxide $\left(\mathrm{OsO}_{4}\right)$ and transferred to pure acetone through an acetone/distilled water series. Specimens were critical point dried in liquid $\mathrm{CO}_{2}$, mounted on stubs, gold-plated under vacuum to a thickness of $200 \AA$ in an Agar High Resolution Sputter Coater Model 20, and examined in a Hitachi S-4300 SEM at an accelerating voltage of $5 \mathrm{kV}$.

Morphometric characters applied herein and their abbreviations are as defined for Cephalobidae in De Ley et al. (1999); terminology of the labial and cephalic region and stoma follows that of Holovachov et al. (2009). Type specimens are deposited in the invertebrate collections of the Department of Zoology, Swedish Museum of Natural History, Stockholm, Sweden (SMNH).

\section{Results}

Phylum Nematoda Diesing, 1861

Class Chromadorea Inglis, 1983

Order Rhabditida Chitwood, 1933

Family Cephalobidae Filipjev, 1934

Genus Paracrobeles Heyns, 1968

\section{Type species}

Paracrobeles laterellus Heyns, 1968

Diagnosis (emended after Holovachov et al. 2009)

Cuticle annulated, without distinctly annulated internal layer; annuli with longitudinal striation (tessellated). Lateral field with two wings (three or four incisures), ending near tail terminus in females and in males. Lip region weakly offset, consisting of six lips arranged in three pairs: one dorsal and two subventral. Pairs of lips separated by primary axils with one or two acute triangular guarding processes; secondary axils shallow. Cephalic probolae with three or four long and slender tines. Labial probolae deeply bifurcated without tines along the slender prongs. Six outer labial and four cephalic papilliform sensilla arranged in a cephaloboid manner. Amphidial aperture rounded, located on lateral lips. Stoma divided into cheilo-, gymno- and stegostom: cheilostom barrel-shaped, with strongly sclerotized bacilliform cheilorhabdia; gymnostom narrow tubular, as wide as stegostom, with weakly sclerotized plate-like gymnorhabdia; stegostom consists of a funnel-shaped prostegostom and variably shaped mesostego-, metastego- and telostegostom parts. Metastegostom tooth absent or present. Pharynx cephaloboid: pharyngeal procorpus cylindrical; metacorpus elongate bulbous; lumen of metacorpus often expanded to a large triradiate chamber with seemingly sclerotized lining; isthmus narrower than metacorpus; basal pharyngeal bulb oval, with strongly developed valves. Nerve ring encircling metacorpus, metacorpus-isthmus junction or 
anterior part of isthmus. Excretory pore opens at level of nerve ring. Deirids present. Female reproductive system cephaloboid; posterior part of ovary straight and relatively short; spermatheca present; postvulval uterine sac present; vulva flat with contour of body or in a depression; vagina straight or directed anteriad. Male reproductive system cephaloboid; spicules cephaloboid, with corpus and manubrium of approximately equal width; gubernaculum plate-like; cornua crurum absent. Male genital papillae: two ventrosublateral pairs located anterior to cloaca; one ventrosublateral pair located just posterior to cloacal opening; two pairs located at middle of tail length; and three pairs (lateral, subventral and subdorsal) near tail terminus; there is a midventral papilla on anterior cloacal lip. Rectum short (about as long as anal body diameter). Phasmid openings located at about one-third to half of tail length in both sexes. Female tail conoid, straight or slightly arcuate ventrad, tail terminus pointed or finely rounded; male tail conoid, slightly arcuate ventrad, tail terminus finely rounded.

\section{Valid species}

P. deserticola Abolafia, Divsalar, Panahi \& Shokoohi, 2014

P. laterellus Heyns, 1968

P. mojavicus Taylor, Baldwin \& Mundo-Ocampo, 2004

P. psammophilus Navarro \& Lluch, 1999

Paracrobeles kelsodunensis sp. nov. urn:1sid:zoobank.org:act:3D3142CA-44FA-422F-9B6A-8A988B3B75EC

Figs 1-2, 3A-C, I; Table 1

\section{Diagnosis}

Paracrobeles kelsodunensis sp. nov. is characterised by a body length of $469-626 \mu \mathrm{m}$ in females and 463-569 $\mu \mathrm{m}$ in males; cuticle coarsely annulated, annuli 3.0-4.8 $\mu \mathrm{m}$ wide at midbody; lateral field with two wings, each separated by a broad groove, extending almost to tail terminus; three pairs of asymmetrical lips, pairs of lips separated by U-shaped primary axils, with two long guarding processes emerging from the first annule, each lip with four (usually) or three (rarely) tines along its margin: two long acute, with or without one shorter in the middle, and one long acute extending along the secondary axil; three long labial probolae, deeply bifurcated, with slender prongs without tines; metastegostom with a strong, anteriorly directed dorsal tooth; pharynx corpus anteriorly spindle-shaped, posteriorly elongate bulbous with dilated lumen; isthmus narrow, demarcated by a break in muscular tissue: anteriorly with heavy musculature, posteriorly further narrowing with reduced musculature; pharyngeal corpus 2.53.3 times isthmus length; nerve ring and excretory pore at level of metacorpus to metacorpus-isthmus junction; spermatheca $24-87 \mu \mathrm{m}$ long; postvulval uterine sac 60-133 $\mu \mathrm{m}$ long; vulva in a depression; spicules 33-38 $\mu \mathrm{m}$ long; and male tail with a 5-8 $\mu \mathrm{m}$ long mucro.

\section{Etymology}

The new species name refers to the place where it was found.

\section{Material examined}

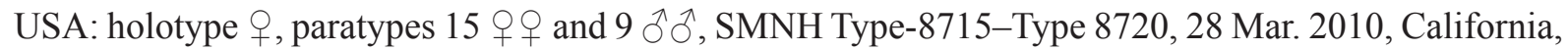
Mojave National Preserve, Kelso Dunes, soil around roots of desert plants $\left(34^{\circ} 53.698^{\prime}\right.$ N, $115^{\circ} 42.155^{\prime}$ W; $34^{\circ} 53.754^{\prime} \mathrm{N}, 115^{\circ} 42.248^{\prime} \mathrm{W}$ and $\left.34^{\circ} 54.226^{\prime} \mathrm{N}, 115^{\circ} 42.200^{\prime} \mathrm{W}\right)$, legit O. Holovachov \& P. De Ley.

\section{Description}

\section{Adult}

Body variably arcuate when killed by heat. Cuticle coarsely annulated, annuli 3.0-4.8 $\mu \mathrm{m}$ wide at midbody. Irregular longitudinal striae give the cuticle a tiled appearance. Lateral field with two wings, 
areolated, each separated by a broad groove, appearing as four incisures under LM, occupying about $20 \%$ of body diameter, extending almost to tail terminus in both sexes. Lip region weakly offset, carrying $6+4$ papillae and two rounded amphid apertures. Three pairs of asymmetrical lips, one dorsal and two ventrolateral. Pairs of lips separated by U-shaped primary axils, with two long guarding processes emerging from the first annule. Each lip with four (commonly) or three (rarely - seen in one specimen so far) tines along its margin: two long acute, with or without one shorter in the middle, and one long acute extending along the secondary axil. Three labial probolae, 13.0-15.5 $\mu \mathrm{m}$ long, deeply bifurcated, with slender prongs without tines. Stoma about one lip region diameter long. Stomatal parts not clearly discernible. Cheilorhabdia oval in latero-median view; metastegostom with a strong, anteriorly directed dorsal tooth. Pharyngeal corpus anteriorly spindle-shaped, posteriorly elongate bulbous with dilated lumen; isthmus narrow, demarcated by a break in muscular tissue: anteriorly with heavy musculature, posteriorly further narrowing with reduced musculature; bulb oval, with valves. Nerve ring and excretory pore vary in position, from the level of metacorpus to metacorpus-isthmus junction, at $60-68 \%$ of neck length and at $57-68 \%$ of neck length, respectively. Deirids at level of isthmus, at $69-82 \%$ of neck length. Excretory canal cuticularised distally.

\section{Female}

Reproductive system monodelphic, prodelphic, in dextral position in relation to intestine. Ovary reflexed posteriorly at oviduct, ovary straight posterior to vulva. Spermatheca well developed. Postvulval uterine sac large, 1.4-3.1 times vulval body diameter (VBD) long. Vagina straight and perpendicular to body axis, about one-third to two-fifths of VBD. Vulva in a sunken area. Intra-uterine eggs 43-66 x 30-36 $\mu \mathrm{m}$. Tail conoid, generally slightly curved ventrad, curved dorsad in some specimens, with 16-21 ventral annuli, non-annulated in terminal 6-12 $\mu \mathrm{m}$, terminus minutely rounded. Rectum sigmoid, about half of ABD long. Phasmid openings located at about one-third to two-fifths of tail length.

\section{Male}

Similar to female in most respects, except for the sexual characters. Reproductive system monorchic, dextral in position; testis reflexed ventrad anteriorly. Spicules slender, paired and symmetrical, strongly curved ventrad; with oval manubrium and subcylindrical, gradually narrowing shaft. Gubernaculum plate-like. Genital papillae distributed as follows: two pairs ventrosublateral precloacal (at 5-11 $\mu \mathrm{m}$ and at 45-57 $\mu \mathrm{m}$ anterior to cloaca), one pair ventrosublateral adcloacal, a single midventral on anterior cloacal lip; two pairs (one ventrosublateral and one lateral) at midtail; three pairs (one lateral, one subventral and one dorsosublateral) near tail terminus. Phasmid openings located at about two-fifths of tail length, one to two annuli posterior to the lateral midtail papillae. Tail slightly curved ventrad, conoid, with a $5-8 \mu \mathrm{m}$ long mucro and minutely rounded terminus.

\section{Remarks}

The population of Paracrobeles kelsodunensis sp. nov. from Kelso Dunes described here agrees in many respects with the description of $P$. mojavicus collected from sandy soil in a lava field, Mojave Desert, California. The new species differs from $P$. mojavicus by having a prominent, anteriorly directed, dorsal metastegostom tooth (vs no metastegostom tooth); a somewhat more anterior position of the excretory pore (at level of metacorpus or metacorpus-isthmus junction vs at level of isthmus); male tail with a 5-8 $\mu \mathrm{m}$ long mucro ( $v s$ male tail without mucro). Type specimens of $P$. mojavicus were examined (see Fig. 3D-I) and among them, one male (Fig. 3I) was found to have a prominent metastegostom tooth. The presence of a metastegostom tooth has not been described for any other species than $P$. kelsodunensis sp. nov., which might indicate that the population described by Taylor et al. (2004) is a mixture of species. One possible explanation could be a polymorphism based on food sources, and a comparison between molecular characters would probably be needed to resolve the status of the Paracrobeles populations from the Mojave Desert. 


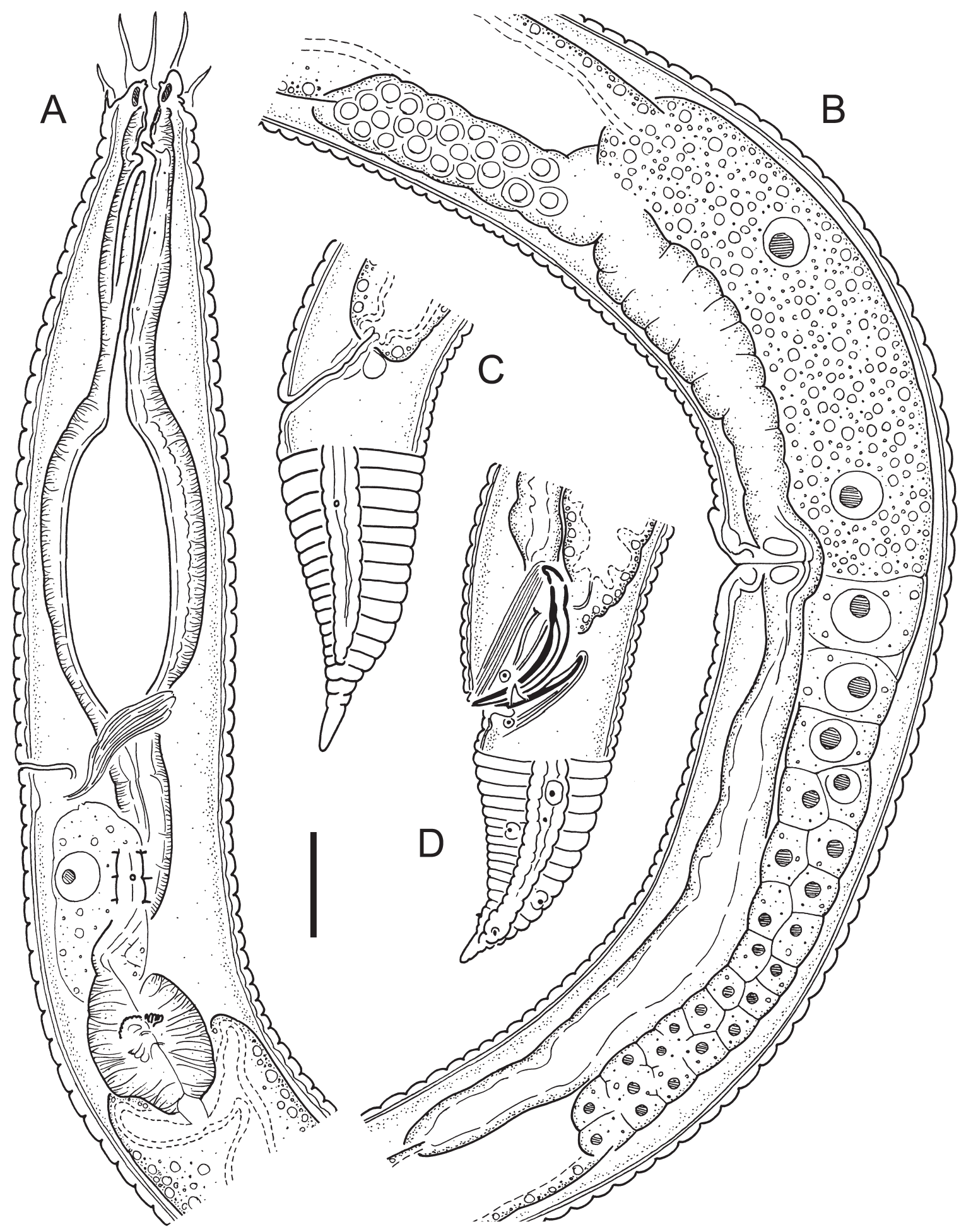

Fig. 1. Paracrobeles kelsodunensis sp. nov. A. Pharyngeal region. B. Female gonad. C. Female tail. D. Male tail. Scale bar $=20 \mu \mathrm{m}$. 

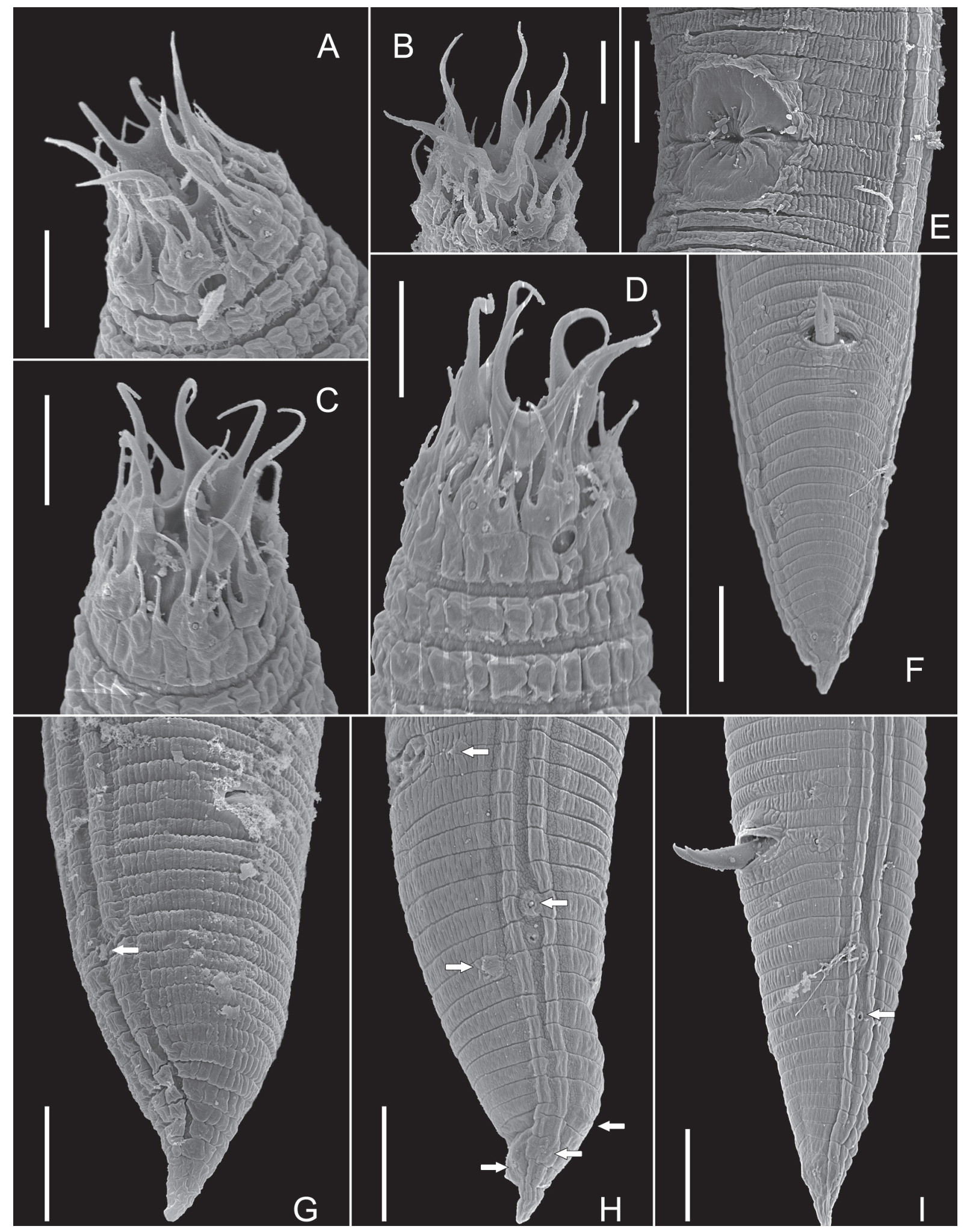

Fig. 2. Paracrobeles kelsodunensis sp. nov. SEM micrographs. A. Anterior end, left lateral view. B. Anterior end, semi-en face view. C-D. Anterior end, subventral view. E. Vulval region. F. Male tail, ventral view. G. Female tail, right sublateral view (arrow points at phasmid). H. Male tail, left lateral view (arrows point at papillae). I. Male tail, left lateral view (arrow points at phasmid). Scale bars: $\mathrm{A}-\mathrm{D}=5 \mu \mathrm{m}, \mathrm{E}-\mathrm{I}=10 \mu \mathrm{m}$. 


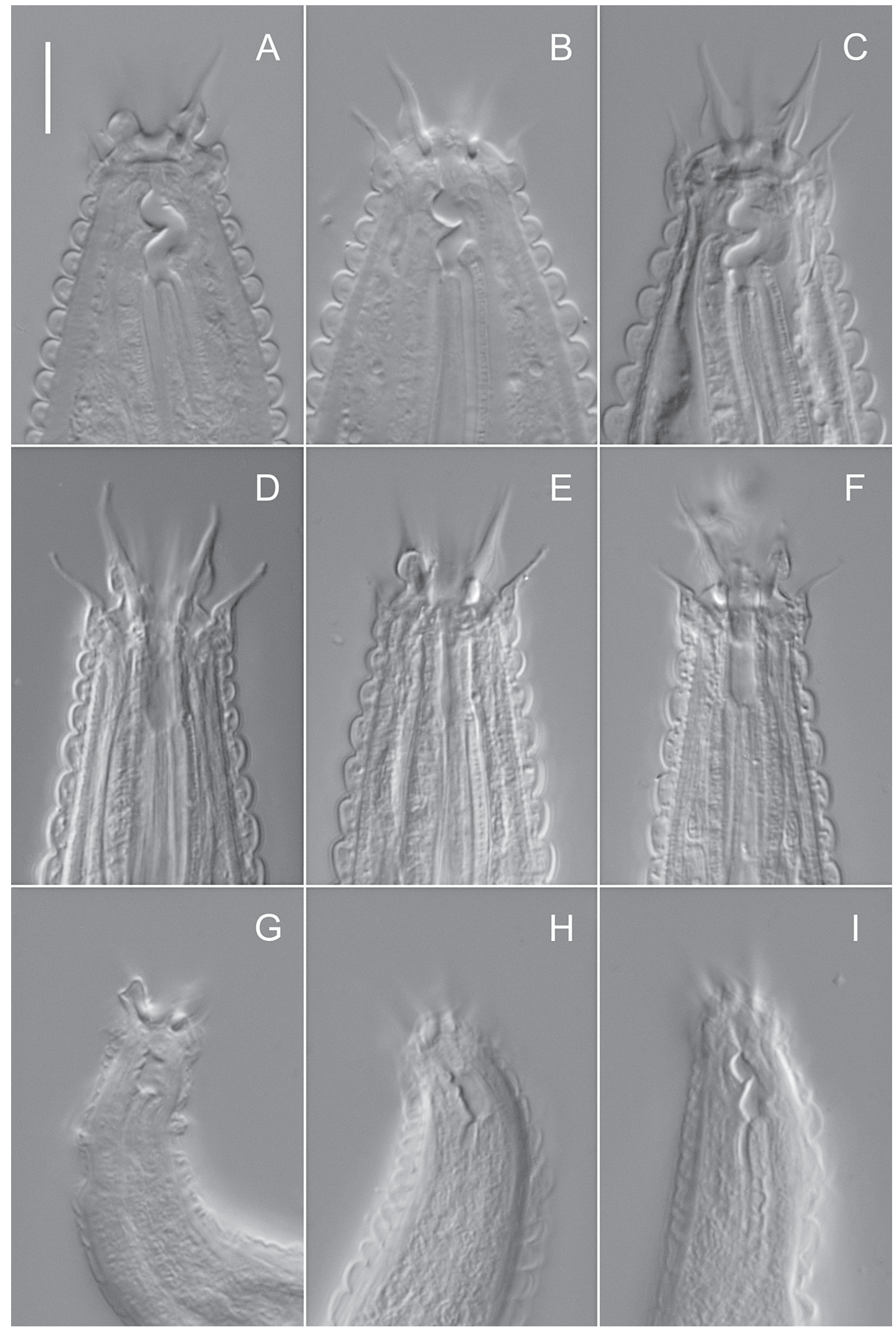

Fig. 3. A-C. Paracrobeles kelsodunensis sp. nov. LM micrographs. A. Male anterior end, ventral side to the right. B-C. Female anterior end, ventral side to the right. D-H. Paracrobeles mojavicus Taylor, Baldwin \& Mundo-Ocampo, 2004. D-F. Female anterior end, ventral side to the right. G-H. Male anterior end, ventral side to the right. I. Paracrobeles cf. kelsodunensis sp. nov., male anterior end, ventral side to the right. Scale bar: $\mathrm{A}-\mathrm{I}=10 \mu \mathrm{m}$. 
Table 1. Measurements (in $\mu \mathrm{m}$ ) of Paracrobeles kelsodunensis sp. nov. from Kelso Dunes, Mojave Desert, California (presented as mean \pm s.d. and (range) or only range). * Number of annuli from anterior end to nerve ring, excretory pore and deirid, respectively; - indicates that data is not applicable.

\begin{tabular}{|c|c|c|c|}
\hline & Holotype $q$ & $\mathbf{1 6}+q_{+}$(incl. holotype) & $9 \hat{\partial}$ \\
\hline Body length & 626 & $538 \pm 51(469-626)$ & $514 \pm 33(463-569)$ \\
\hline Body diameter (BD) & 42 & $43.0 \pm 3.2(37-49)$ & $37.5 \pm 2.3(34-41)$ \\
\hline Pharynx length & 185 & $169 \pm 11.8(150-188)$ & $161.5 \pm 9.5(147-173)$ \\
\hline Tail length & 66 & $54.5 \pm 6.4(42-66)$ & $54.0 \pm 3.9(47-58)$ \\
\hline Anal or cloacal diameter (ABD) & 29 & $27.6 \pm 1.7(25-31)$ & $29.9 \pm 0.9(28-31)$ \\
\hline Vulva or Testis & 391 & $334 \pm 29.1(297-391)$ & $265 \pm 31(223-315)$ \\
\hline V-A/T & 2.8 & $2.8 \pm 0.3(2.5-3.4)$ & - \\
\hline $\mathbf{a}$ & 14.9 & $12.5 \pm 1.0(10.9-14.9)$ & $13.7 \pm 1.2(12.1-16.7)$ \\
\hline b & 3.4 & $3.2 \pm 0.2(2.9-3.7)$ & $3.2 \pm 0.2(2.9-3.6)$ \\
\hline c & 9.5 & $10.0 \pm 0.6(9.1-11.5)$ & $9.6 \pm 1.0(8.0-11.8)$ \\
\hline$c^{\prime}$ & 2.3 & $2.0 \pm 0.2(1.6-2.3)$ & $1.8 \pm 0.2(1.6-2.1)$ \\
\hline $\mathrm{V}$ or $\mathrm{T}(\%)$ & 62.5 & $62.0 \pm 1.2(60-65)$ & $51.1 \pm 5.1(44-59)$ \\
\hline Lip region diameter & 18 & $17.7 \pm 0.4(17-18)$ & $17.1 \pm 0.6(16-18)$ \\
\hline Labial probolae & 15.5 & $14.7 \pm 0.7(13.0-15.5)$ & $14.1 \pm 0.6(13.0-14.5)$ \\
\hline Stoma length & 14.5 & $14.9 \pm 0.5(14.5-15.5)$ & $15.1 \pm 0.5(14.5-15.5)$ \\
\hline Corpus length & 114 & $106.1 \pm 5.3(100-119)$ & $99.7 \pm 3.9(94-105)$ \\
\hline Isthmus length & 39 & $35.9 \pm 3.5(30-42)$ & $33.4 \pm 2.3(29-37)$ \\
\hline Bulb length & 30 & $29.6 \pm 1.2(28-33)$ & $29.9 \pm 1.0(28-31)$ \\
\hline Bulb diameter & 22 & $22.3 \pm 1.0(20-23)$ & $21.0 \pm 0.9(20-22)$ \\
\hline Corpus/isthmus ratio & 2.9 & $3.0 \pm 0.3(2.5-3.3)$ & $3.0 \pm 0.2(2.7-3.3)$ \\
\hline Nerve ring from anterior end & 133 & $123.9 \pm 7.7(112-142)$ & $119.0 \pm 8.0(109-137)$ \\
\hline Excretory pore from anterior end & 141 & $119.4 \pm 10.4(102-142)$ & $114.8 \pm 10.8(101-133)$ \\
\hline Deirid from anterior end & 161 & $152.8 \pm 11.1(135-163)$ & $134.0 \pm 13.4(110-155)$ \\
\hline $\mathbf{R}_{\mathrm{NR}}^{*}$ & 24 & $26.1 \pm 1.4(23-29)$ & $26.4 \pm 1.2(24-28)$ \\
\hline $\mathbf{R}_{\mathrm{EP}} *$ & 26 & $24.9 \pm 1.3(22-27)$ & $25.3 \pm 1.2(24-27)$ \\
\hline $\mathbf{R}_{\mathrm{DEI}}^{*}$ & 31 & $31.8 \pm 2.2(29-36)$ & $30.8 \pm 2.2(27-34)$ \\
\hline Annuli width at midbody & $4.0-4.8$ & $3.0-4.8$ & $3.4-4.0$ \\
\hline Annuli width anteriorly & $4.0-4.8$ & $3.4-4.8$ & $3.0-4.0$ \\
\hline Vagina or Testis flexure length & 14.5 & $13.6 \pm 0.8(13.0-15.5)$ & $47.7 \pm 13.0(31-72)$ \\
\hline Spermatheca or Spicule length & 60 & $49.7 \pm 16.8(24-87)$ & $35.1 \pm 1.5(33-38)$ \\
\hline PUS or Gubernaculum length & 129 & $102.1 \pm 20.4(60-133)$ & $19.9 \pm 1.3(18-22)$ \\
\hline PUS/VBD & 3.1 & $2.6 \pm 0.5(1.4-3.1)$ & - \\
\hline Rectum & 18 & $17.9 \pm 1.3(14.5-20.5)$ & - \\
\hline Rectum/ABD & 0.6 & $0.6 \pm 0.1(0.5-0.7)$ & - \\
\hline Phasmid & 20.5 & $19.1 \pm 1.9(16-29)$ & $21.5 \pm 2.5(18-26)$ \\
\hline Phasmid (\% of tail) & 31 & $35.5 \pm 3.4(31-40)$ & $40.1 \pm 2.8(37-45)$ \\
\hline
\end{tabular}

Key to species of Paracrobeles (emended after Abolafia et al. 2014; when using the key, please also consult Table 2 for additional diagnostic information)

1. Spicules less than $40 \mu \mathrm{m}$ long

- Spicules more than $40 \mu \mathrm{m}$ long, primary axils with single guarding process.

2. Vulva at $58-60 \%$ of body length

P. laterellus Heyns, 1968

- Vulva at $60-71 \%$ of body length, primary axils with two guarding processes

3. Metastegostom tooth absent .

P. mojavicus Taylor, Baldwin \& Mundo-Ocampo, 2004

- Metastegostom tooth present, anteriorly directed

P. kelsodunensis sp. nov. 
BOSTRÖM S. \& HOLOVACHOV O., New species of Paracrobeles from Kelso Dunes

\begin{tabular}{|c|c|c|c|c|c|c|c|c|c|c|c|c|c|}
\hline 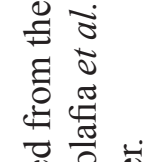 & 离 & \multicolumn{2}{|c|}{ 匟 } & \multicolumn{2}{|c|}{ 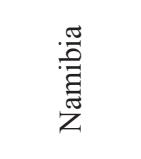 } & 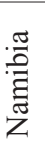 & 总泀 & \multicolumn{2}{|c|}{$\begin{array}{l}\text { : } \\
\text { की }\end{array}$} & \multicolumn{2}{|c|}{$\frac{\lambda}{\Phi}$} & \multicolumn{2}{|c|}{ 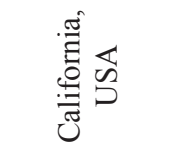 } \\
\hline 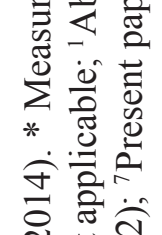 & 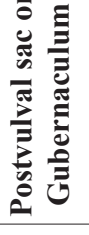 & 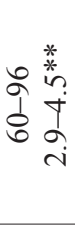 & 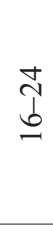 & 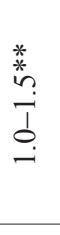 & $\begin{array}{l}\text { Iิ } \\
\underline{I}\end{array}$ & \& & 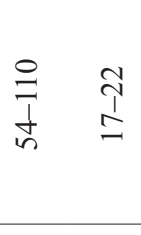 & $\frac{\overrightarrow{0}}{\frac{1}{n}}$ & 仓े & $\begin{array}{l}8 \\
\vdots \\
1 \\
6\end{array}$ & $\begin{array}{l}\overrightarrow{0} \\
\infty \\
\sim\end{array}$ & $\begin{array}{l}m \\
0 \\
\delta\end{array}$ & $\begin{array}{l}\text { ה } \\
\infty \\
\infty\end{array}$ \\
\hline 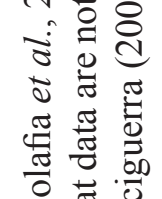 & 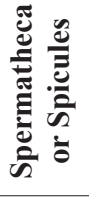 & $\begin{array}{l}n \\
m \\
m\end{array}$ & $\begin{array}{l}0 \\
b \\
b\end{array}$ & 1 & 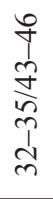 & $\underset{\sim}{2}$ & $\begin{array}{cc}\hat{A} & \hat{0} \\
\infty & \infty \\
\infty & \infty\end{array}$ & $\stackrel{*}{\stackrel{*}{+}}$ & $\begin{array}{l}\tilde{i} \\
\text { y }\end{array}$ & $\hat{n}$ & $\begin{array}{l}\vec{\infty} \\
\infty \\
\infty\end{array}$ & $\begin{array}{l}\infty \\
\stackrel{d}{d}\end{array}$ & $\underset{m}{\infty}$ \\
\hline 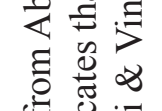 & 馬 & $\begin{array}{l}\hat{6} \\
\hat{n} \\
\hat{n}\end{array}$ & $\begin{array}{l}\dot{+} \\
\dot{\infty} \\
\dot{+}\end{array}$ & $\begin{array}{l}8 \\
0 \\
0 \\
\text { in }\end{array}$ & $\begin{array}{l}n \\
i \\
i\end{array}$ & in & $\begin{array}{ll}8 & \overline{0} \\
1 & \vdots \\
\gamma & f\end{array}$ & $\begin{array}{l}n \\
b \\
b\end{array}$ & $\begin{array}{c}\hat{n} \\
\stackrel{\sim}{q}\end{array}$ & $\begin{array}{l}\infty \\
0 \\
\infty \\
i\end{array}$ & $\begin{array}{l}\stackrel{0}{b} \\
b \\
i n\end{array}$ & $\begin{array}{l}8 \\
\stackrel{1}{1} \\
\mathcal{Y}\end{array}$ & $\begin{array}{c}\infty \\
\frac{1}{\gamma} \\
\frac{1}{\gamma}\end{array}$ \\
\hline 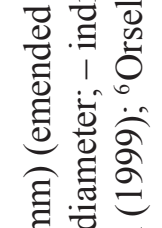 & 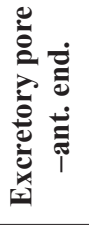 & $\begin{array}{l}\exists \\
\bar{b} \\
\varnothing\end{array}$ & $\frac{\stackrel{1}{1}}{1}$ & $\stackrel{*}{\stackrel{*}{\circ}}$ & 1 & $\underline{\underline{0}}$ & $\begin{array}{ll}\hat{b} & 8 \\
1 & 1 \\
n & 0 \\
= & 0\end{array}$ & $\begin{array}{l}\bar{\Xi} \\
\text { ò }\end{array}$ & 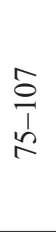 & $\begin{array}{l}\stackrel{\Xi}{\emptyset} \\
\stackrel{+}{\Xi}\end{array}$ & 1 & 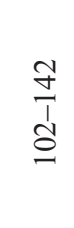 & $\frac{m}{1}$ \\
\hline 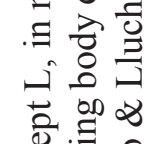 & 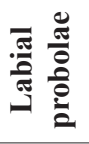 & $\stackrel{0}{ \pm}$ & $\begin{array}{l}0 \\
-1 \\
n\end{array}$ & $\stackrel{*}{\stackrel{*}{0}}$ & 1 & \% & 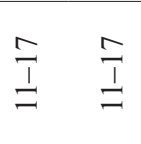 & $\frac{1}{0}$ & 1 & $\stackrel{\infty}{I}$ & $\stackrel{1}{m}$ & $\begin{array}{l}0 \\
\cdots \\
-1\end{array}$ & $\frac{n}{n}$ \\
\hline 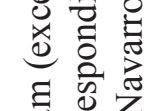 & 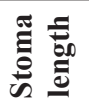 & $\stackrel{ \pm}{I}$ & $\begin{array}{l} \pm \\
\beth\end{array}$ & * & I & $\simeq$ & $\begin{array}{ll}\infty & \infty \\
\frac{1}{n} & n\end{array}$ & $\stackrel{ \pm}{d}$ & $\frac{ \pm}{d}$ & $\frac{\infty}{1}$ & 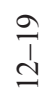 & $\begin{array}{l}0 \\
\pm \\
\pm\end{array}$ & $\frac{0}{ \pm}$ \\
\hline 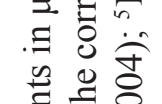 & $\stackrel{\sigma}{0}^{0}$ & $\begin{array}{l}0 \\
0 \\
0 \\
n\end{array}$ & | & $\begin{array}{l}8 \\
0 \\
\infty \\
n\end{array}$ & 1 & in & $\begin{array}{l}\vec{i} \\
\text { d̂ }\end{array}$ & $\begin{array}{l}8 \\
0 \\
0\end{array}$ & 1 & $\begin{array}{l}\text { So } \\
\text { ô }\end{array}$ & 1 & $\begin{array}{l}8 \\
0 \\
8 \\
8\end{array}$ & 1 \\
\hline 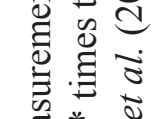 & is & $\begin{array}{l}0 \\
\dot{T} \\
0 \\
i\end{array}$ & $\stackrel{\tilde{\omega}}{\infty}$ & $\stackrel{*}{i}$ & $\begin{array}{l}\text { * } \\
\text { i }\end{array}$ & $\tilde{i}$ & 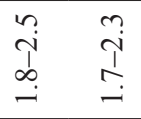 & $\begin{array}{l}\hat{i} \\
\hat{i}\end{array}$ & 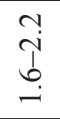 & $\begin{array}{l}\infty \\
i \\
i \\
i \\
i\end{array}$ & $\begin{array}{l}\infty \\
i \\
i \\
\end{array}$ & 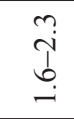 & $\begin{array}{l}\vec{i} \\
\stackrel{i}{-}\end{array}$ \\
\hline 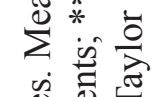 & u & $\frac{0}{a}$ & $\frac{m}{a}$ & $\begin{array}{l}n \\
I\end{array}$ & $\stackrel{I}{I}$ & $=$ & $\begin{array}{ll}m & \frac{1}{a} \\
a & 0\end{array}$ & $=$ & $\frac{a}{a}$ & $\frac{0}{\infty}$ & $\hat{\infty}$ & $\frac{1}{a}$ & $\stackrel{1}{\infty}$ \\
\hline 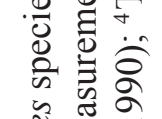 & $=$ & $\begin{array}{l}\dot{0} \\
\dot{T} \\
\dot{0} \\
\dot{m}\end{array}$ & $\begin{array}{l}0 \\
\dot{+} \\
\dot{m} \\
\dot{r}\end{array}$ & $\begin{array}{l}F \\
\dot{+} \\
m \\
m\end{array}$ & $\begin{array}{l}a \\
\dot{n} \\
\dot{n}\end{array}$ & $\hat{m}$ & 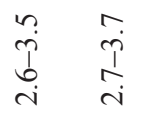 & $\begin{array}{r}\hat{j} \\
\hat{i}\end{array}$ & $\begin{array}{l}\dot{0} \\
\dot{p} \\
\dot{m}\end{array}$ & $\begin{array}{l}\hat{i} \\
\hat{i} \\
\hat{i}\end{array}$ & $\begin{array}{l}i \\
\dot{j} \\
i\end{array}$ & $\begin{array}{l}\hat{j} \\
\hat{i}\end{array}$ & $\begin{array}{l}0 \\
\dot{j} \\
\text { aे }\end{array}$ \\
\hline 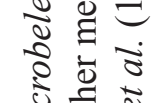 & $\pi$ & $\stackrel{\infty}{J}$ & $\frac{a}{a}$ & & 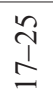 & $\stackrel{\infty}{-}$ & 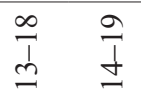 & $\underline{a}$ & $\frac{\infty}{1}$ & $\begin{array}{l}0 \\
m \\
-1\end{array}$ & 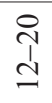 & $\stackrel{n}{=}$ & $\stackrel{I}{\simeq}$ \\
\hline 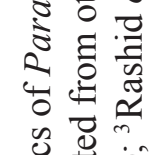 & ב & $\begin{array}{l}8 \\
0 \\
0 \\
\infty \\
+ \\
0\end{array}$ & $\begin{array}{l}n \\
0 \\
0 \\
0 \\
0 \\
+ \\
0\end{array}$ & 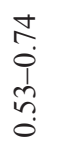 & $\begin{array}{l}0 \\
0 \\
0 \\
1 \\
n \\
0\end{array}$ & $\begin{array}{l}n \\
\tilde{n} \\
0\end{array}$ & 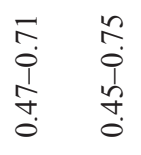 & $\begin{array}{l}n \\
n \\
0 \\
b \\
n \\
0\end{array}$ & 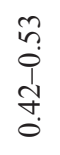 & $\begin{array}{l}\infty \\
n \\
0 \\
\vdots \\
+ \\
0\end{array}$ & $\begin{array}{l}\vec{\sigma} \\
0 \\
1 \\
n \\
n \\
0\end{array}$ & 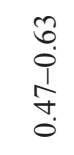 & $\begin{array}{l}n \\
n \\
0 \\
b \\
0 \\
0\end{array}$ \\
\hline 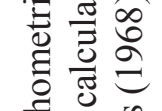 & $=$ & $\begin{array}{l}\text { O+ } \\
\text { O+ } \\
\infty\end{array}$ & $\begin{array}{l}\gamma_{0} \\
<0 \\
\infty\end{array}$ & $\begin{array}{l}\text { O+ } \\
\text { O+ } \\
\forall\end{array}$ & $\begin{array}{l}50 \\
80 \\
0\end{array}$ & O+ & $\begin{array}{ll}\text { O+ } & \text { so } \\
\text { O+ } & 8 \\
= & 9 \\
= & 9\end{array}$ & $\begin{array}{l}\text { O+ } \\
\text { O+ } \\
\text { ○ }\end{array}$ & $\begin{array}{l}r_{0} \\
8 \\
\circ \\
0\end{array}$ & $\begin{array}{l}\text { O+ } \\
\text { Ot } \\
\text { t }\end{array}$ & $\begin{array}{l}\text { f } \\
\text { fo } \\
0\end{array}$ & $\begin{array}{l}\text { O+ } \\
\text { O+ } \\
\text { O } \\
-\end{array}$ & $\begin{array}{l}\text { so } \\
\text { so } \\
\sigma\end{array}$ \\
\hline 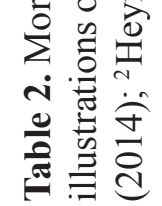 & 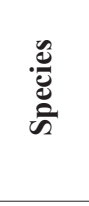 & & & & & בั้ & 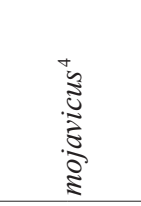 & & & & & & \\
\hline
\end{tabular}


4. Lips with three tines (including the ones in secondary axils); postvulval sac not swollen

P. deserticola Abolafia, Divsalar, Panahi \& Shokoohi, 2014

- Lips with four tines (including the ones in secondary axils); postvulval sac swollen

P. psammophilus Navarro \& Lluch, 1999

\section{Discussion}

The Mojave Desert and especially the Kelso Dunes seem to be an area with a high diversity of species of the family Cephalobidae Filipjev, 1934. Representatives of this family are mostly terrestrial and bacteria-consuming nematodes with a worldwide distribution. They seem to be especially diverse and abundant in deserts, and sand dunes appear to be a suitable habitat for cephalobids. About ten new species have been described from the Mojave Desert, which might indicate a hot spot for cephalobid diversity (De Ley 2014). Hitherto one new genus and six new species, including the one described here, and one already known species have been described from the Kelso Dunes (Boström \& Holovachov 2012, 2013a, 2013b, 2014). Some of the new species described from the Mojave Desert are closely related, which might further indicate that speciation processes are going on in this area. Species of the genus Paracrobeles are rare inhabitants of terrestrial ecosystems and have a rather restricted distribution. So far they have been found in warm, dry sandy soils in southern Africa (South Africa and Namibia), the Mediterranean (Spain and Italy), California and Iran (Abolafia et al. 2014; Heyns 1968; Navarro \& Lluch 1999; Orselli \& Vinciguerra 2002; Rashid et al. 1990; Taylor et al. 2004). The description of the new species adds morphological data important for species identification and broadens the diagnosis of Paracrobeles.

\section{Acknowledgements}

The second author was supported in part by an award from the NSF Partnerships for Enhancing Expertise in Taxonomy (PEET) program grant "PEET: Training the Next Generation of Nematode Taxonomists: Applying the Tools of Modern Monography Across Free-living and Parasitic Tylenchina" (DEB-0731516). Sampling was performed by O. Holovachov and P. De Ley under permit \# MOJA2010-SCI-0003 from the National Park Service, United States Department of Interior.

\section{References}

Abolafia J., Divsalar N., Panahi H. \& Shokoohi E. 2014. Description of Paracrobeles deserticola sp. n. and Nothacrobeles hebetocaudatus sp. n. (Nematoda: Rhabditida: Cephalobidae) from Iran and the phylogenetic relationships of these two species. Zootaxa 3827 (1): 1-19. http://dx.doi.org/10.11646/ zootaxa.3827.1.1

Boström S. \& Holovachov O. 2012. Description of Chilodellus eremus gen. n., sp. n. and Stegelleta arenaria sp. n. (Rhabditida: Cephalobidae) from Kelso Dunes, Mojave National Preserve, California, USA. Journal of Nematode Morphology and Systematics 15 (1): 21-31.

Boström S. \& Holovachov O. 2013a. Description of two new species of Nothacrobeles Allen \& Noffsinger, 1971 (Rhabditida: Cephalobidae) from Kelso Dunes, Mojave National Preserve, California, USA. Journal of Nematode Morphology and Systematics 16 (1): 25-34.

Boström S. \& Holovachov O. 2013b. Description of one new species of Heterocephalobellus Rashid, Geraert \& Sharma, 1985 (Rhabditida, Cephalobidae) from Kelso Dunes, Mojave National Preserve, California, USA and Monte desert, Usno, Argentina. Journal of Nematode Morphology and Systematics 16 (2): 161-166.

Boström S. \& Holovachov O. 2014. Descriptions of species of Stegelleta Thorne, 1938 (Nematoda, Rhabditida, Cephalobidae) from California, New Zealand and Senegal, and a revision of the genus. European Journal of Taxonomy 87: 1-19. http://dx.doi.org/10.5852/ejt.2014.87 
De Ley I.T., De Ley P., Baldwin J.G., Mundo-Ocampo M. \& Nadler S.A. 1999. Three new species of Nothacrobeles (Nemata: Cephalobidae) from the Mojave Desert, California. Journal of Nematology 31 (4): 482-497.

De Ley P. 2014. Past and present highlights of nematode research in the Mojave Desert. Mojave National Preserve Science Newsletter May 2014: 5-10.

Heyns J. 1968. Paracrobeles laterellus n. gen., n. sp. from South Africa (Nematoda: Cephalobidae). Nematologica 14: 511-514. http://dx.doi.org/10.1163/187529268X00192

Holovachov O., De Ley I.T., Mundo-Ocampo M. \& De Ley P. 2009. Identification of Cephaloboidea (Nematoda). EUMAINE, Gent and Nematology, UC Riverside. Available from http://www.nrm.se/ download/18.9ff3752132fdaeccb6800015606/CEPHALOBOIDEA.pdf [accessed 14 Jan 2015]

Navarro P. \& Lluch J. 1999. Paracrobeles psammophilus sp. nov. (Nematoda: Cephalobidae) from El Saler, Valencia (Spain). Journal of Zoology 249 (4): 481-485. http://dx.doi.org/10.1111/j.1469-7998.1999. $\underline{\text { tb01220.x }}$

Orselli L. \& Vinciguerra M.T. 2002. Nematodes from Italian sand dunes. 6. Two new and three rare species of Cephalobidae (Nematoda). Nematologia mediterranea 30: 211-220.

Rashid F., Heyns J. \& Coomans A.1990. Paracrobeles and Acrobeles species from South West Africa/ Namibia with description of a new Acrobeles species (Nematoda: Cephalobidae). Phytophylactica 22 (1): 41-49.

Taylor T.M., Baldwin J.G. \& Mundo-Ocampo M. 2004. Paracrobeles mojavicus sp. n. (Nematoda: Cephalobidae) from the Mojave Desert, California. Journal of Nematode Morphology and Systematics 6(2): 151-160.

Manuscript received: 9 December 2014

Manuscript accepted: 15 January 2015

Published on: 23 March 2015

Topic editor: Rudy Jocqué

Desk editor: Charlotte Thionois

Printed versions of all papers are also deposited in the libraries of the institutes that are members of the EJT consortium: Muséum national d'Histoire naturelle, Paris, France; Botanic Garden Meise, Belgium; Royal Museum for Central Africa, Tervuren, Belgium; Natural History Museum, London, United Kingdom; Royal Belgian Institute of Natural Sciences, Brussels, Belgium; Natural History Museum of Denmark, Copenhagen, Denmark. 